\title{
Scaling properties of velocity and temperature spectra above the surface friction layer in a convective atmospheric boundary layer
}

\author{
K. G. McNaughton, R. J. Clement, and J. B. Moncrieff \\ School of GeoSciences, The University of Edinburgh, Edinburgh EH9 3JU, Scotland \\ Received: 28 August 2006 - Revised: 8 January 2007 - Accepted: 18 May 2007 - Published: 11 June 2007
}

\begin{abstract}
We report velocity and temperature spectra measured at nine levels from 1.42 meters up to $25.7 \mathrm{~m}$ over a smooth playa in Western Utah. Data are from highly convective conditions when the magnitude of the Obukhov length (our proxy for the depth of the surface friction layer) was less than $2 \mathrm{~m}$. Our results are somewhat similar to the results reported from the Minnesota experiment of Kaimal et al. (1976), but show significant differences in detail. Our velocity spectra show no evidence of buoyant production of kinetic energy at at the scale of the thermal structures. We interpret our velocity spectra to be the result of outer eddies interacting with the ground, not "local free convection".

We observe that velocity spectra represent the spectral distribution of the kinetic energy of the turbulence, so we use energy scales based on total turbulence energy in the convective boundary layer (CBL) to collapse our spectra. For the horizontal velocity spectra this scale is $\left(z_{i} \epsilon_{o}\right)^{2 / 3}$, where $z_{i}$ is inversion height and $\epsilon_{o}$ is the dissipation rate in the bulk CBL. This scale functionally replaces the Deardorff convective velocity scale. Vertical motions are blocked by the ground, so the outer eddies most effective in creating vertical motions come from the inertial subrange of the outer turbulence. We deduce that the appropriate scale for the peak region of the vertical velocity spectra is $\left(z \epsilon_{o}\right)^{2 / 3}$ where $z$ is height above ground. Deviations from perfect spectral collapse under these scalings at large and small wavenumbers are explained in terms of the energy transport and the eddy structures of the flow.

We find that the peaks of the temperature spectra collapse when wavenumbers are scaled using $\left(z^{1 / 2} z_{i}^{1 / 2}\right)$. That is, the lengths of the thermal structures depend on both the lengths of the transporting eddies, $\sim 9 z$, and the progressive aggregation of the plumes with height into the larger-scale structures of the CBL. This aggregation depends, in top-down fash-
\end{abstract}

Correspondence to: K. G. McNaughton

(keith@mcnaughty.com) ion, on $z_{i}$. The whole system is therefore highly organized, with even the smallest structures conforming to the overall requirements of the whole flow.

\section{Introduction}

For modeling purposes convective boundary layers (CBLs) are usually divided into a number of horizontal layers, each defined empirically in terms of the set of similarity parameters needed to reduce observed turbulent statistics to universal relationships (Holtslag and Nieuwstadt, 1986; Kader and Yaglom, 1990). We will be concerned with the layer that lies above the surface friction layer and below the mixed layer in highly convective conditions. Tennekes (1970) has called this the 'local free convection layer'. In it many turbulence statistics have approximately constant values when scaled using the observation height, $z$, and the free-convection velocity scale, $u_{f}=\left(g z H_{0} / T\right)^{1 / 3}$, where $g$ is acceleration due to gravity and $H_{0}$ is the kinematic heat flux at the ground so $H_{0}=\overline{w^{\prime} T^{\prime}}$ where $w$ is vertical wind speed and $T$ is temperature. In this layer heat is transported principally in rising plumes of warm air. These plumes can be detected as the coherent arrival of warmer air at successive levels on towers, first at upper levels then progressively to lower levels down to the ground (Kaimal and Businger, 1970; Kaimal et al., 1976; Webb, 1977).

While the presence of plumes cannot be doubted, the dynamics of this layer might not be local free convection, at least not in the sense of buoyant plumes rising autonomously under the local action of their own buoyancy. Indeed this model creates a number of problems: it does not explain why the scalar quantities seem to obey free convection scaling down to $z /|L| \approx 0.04$, where $L$ is the Obukhov length, at which height the local buoyant production term in the kinetic energy budget equation is only $4 \%$ of the total production; it does not explain why the friction velocity, $u_{*}$,

Published by Copernicus Publications on behalf of the European Geosciences Union and the American Geophysical Union. 
should disappear as a scaling parameter even while the momentum flux, $-u_{*}^{2}$, is approximately constant with height; and, most curiously of all, it does not explain why "free convection scaling" should apply down to lower levels for scalar quantities (e.g. temperature gradient, temperature variance and temperature dissipation rate) than for purely dynamical quantities such as vertical velocity variance and energy dissipation rate (Priestley, 1955; Kader and Yaglom, 1990). Associated with these are questions about the nature of the transition from the surface friction layer - the layer where $u_{*}$ is important in scaling the velocity statistics - to the outer layer. McNaughton (2004b) has shown that buoyancy forces do no work in the surface friction layer because they are opposed by balancing pressure reaction forces. This being so, the transition from an unstable surface friction layer to a free convection layer would have to be a transition from a layer where buoyancy has no net effect to one where local buoyancy forces wholly drive the vertical motions. How would such a transition work?

In this paper we look again at the "local free convection layer". First we review results of (Kaimal et al., 1976), who made turbulence measurements right up through convective boundary layers over flat land in Minnesota (Kaimal and Wyngaard, 1990). We reinterpret these results in terms of the turbulence structure and the full kinetic energy balance of the whole CBL, and we propose a new scaling model for velocity spectra in the CBL. We conclude that the "local free convection layer" is misnamed and that here, as in the surface friction layer, buoyancy does not contribute to the local production of turbulence energy. We then test this interpretation using new turbulence spectra measured over a smooth and extensive playa in Western Utah, USA. Data were selected for convective conditions such that $|L|<z \ll z_{i}$. The new spectra are broadly consistent with those from Minnesota, but compared to them we find the velocity variance to decrease with height both at large wavenumbers and at some smaller wavenumbers. We also find that the temperature spectra have mixed length scales. We argue that these results support and refine our interpretation of the Minnesota results.

\section{Scaling the Minnesota spectra}

The scientific backdrop to our experiment is the concepts and scaling theory of "local free convection", as given by Wyngaard et al. (1971), and the experimental results and analysis from the Minnesota experiment, as reported by Kaimal et al. (1976). Here we review the Minnesota observations and propose a new interpretation based on the kinetic energy balance of the whole CBL. We argue that the Minnesota results show that turbulence in the CBL should be treated as a single selforganizing system, with system-wide dynamics, rather than one where local processes depend on local variables only.
We deduce reference spectra that will serve as a standard of comparison for our own results, presented below in Sect. 5 .

\section{$2.1 \quad$ Scaling $u$ and $v$ spectra}

Reference spectra are defined here as spectra that have the scaling properties reported by Kaimal et al. (1976) for the Minnesota results, extended consistently to vertical velocity spectra. Kaimal et al. collapsed their $u$ (streamwise velocity) and $v$ (transverse velocity) spectra, to universal forms by first non-dimensionalizing their wavenumbers using measured inversion height, $z_{i}$, to match the peak positions, then scaling their energies to match the inertial subranges. Though this is what they did, Kaimal et al. (1976) wrote the scaling factor for their $u$ and $v$ spectra as $w_{*}^{2} \psi^{2 / 3}$, where $w_{*}$ is the Deardorff convection velocity scale given by

$w_{*}=\left(\frac{z_{i} g H_{0}}{T}\right)^{1 / 3}$

(Deardorff, 1970), and $\psi$ is a dimensionless dissipation rate, given by

$\psi=\frac{\epsilon T}{g H_{0}}$

where $\epsilon$ is the dissipation rate at each level. Manipulation on these definitions shows that their axis scale $w_{*}^{2} \psi^{2 / 3}$ can also be written as $\left(z_{i} \epsilon\right)^{2 / 3}$.

In most of their runs Kaimal et al. (1976) found the values of $\epsilon$ above the surface friction layer to be independent of height. The likely reason is that the whole range of small eddies associated with dissipation are swept along within the larger, $z_{i}$-scale eddies in the CBL, and so are well mixed. Whatever the reason, it is apparent that a single value of $\epsilon$ can be used to characterize the dissipation rate at all levels above the surface friction layer in typical CBLs. We shall call this value the "outer" dissipation rate, and write it as $\epsilon_{o}$.

Kaimal et al. (1976) used the reduced frequency, $f z_{i} / U$, on the frequency axis of their Fig. 3, where $U$ is the mean wind at each observation height. On this scale the plotted peaks of the pre-multiplied $u$ and $v$ power spectra move to smaller frequencies as height increases. Kaimal et al. (1976) also note that these peaks occur at the same natural frequency at all heights, reflecting the passage of the same large, CBL-spanning, convective structures over instruments at all heights. The peak positions can be matched using the mean wind in the boundary layer, $U_{m}$, rather than $U$ to construct the frequency scale. Because we are concerned with the shapes and sizes of eddies, we will work with wavenumbers, calculated using Taylor's frozen turbulence assumption and using $U_{m}$ to make the conversions.

When interpreting the Minnesota results from above the surface friction layer we will assume them to be plotted on a wavenumber axis constructed using $U_{m}$, which scale matches the positions of the spectral peaks at all heights, and the inertial subranges then normalized by the average dissipation 
rate above the surface friction layer, $\epsilon_{o}$. This forces the $u$ and $v$ spectra to collapse to a single curve when $\epsilon$ is constant with height.

Notice that this is not way Kaimal et al. (1976) presented their results. They made no assumptions about $\epsilon$ when matching the inertial subranges of their spectra, allowing it to vary with height as the data dictated. They then used their results to discover the constancy of $\epsilon$ with height above the surface friction layer (Kaimal et al. (1976), Fig. 4). We turn this about and adopt the constancy of $\epsilon$ as a basic property of CBLs, and so use $\epsilon_{o}$ as a basic parameter for scaling spectra.

\subsubsection{Interpreting the shapes of $u$ and $v$ spectra}

The shapes of the Minnesota spectra are significant. The premultiplied $u$ and $v$ have single peaks at $\kappa z_{i} \sim 2 \pi$ followed by regions with $-2 / 3$ slopes on log-log plots, which regions extend unbroken from near the peaks up to very high wavenumbers. These $-2 / 3$ regions do not wholly represent inertial sub-ranges, despite their slopes, because this turbulence is influenced by the ground and so far from isotropic where $\kappa z<1$. Even so, the $-2 / 3$ slopes are clearly related to an inertial subrange even where $\kappa z<1$. The Minnesota $u$ and $v$ spectra did not change with height, so the $-2 / 3$ regions nearer the ground were the same as those found at mid levels of the CBL. At mid levels the turbulence can be isotropic from near the peak wavenumbers on upwards, and at that level a $-2 / 3$ slope is diagnostic of a true inertial subrange. Thus the shapes of the near-ground spectra are dictated by Kolmogorov's law for the outer turbulence. Since the nearsurface turbulence is fully characterized by parameters taken from the outer turbulence, we deduce that the near-surface turbulence represents the effects of eddies from the outer inertial subrange impinging onto the ground.

We can then deduce the velocity scale of the near-ground turbulence from the velocity spectra of the turbulence at mid levels in the CBL. We know that inertial subranges are purely transmission regions of power spectra - regions where kinetic energy is neither created nor destroyed. The shape of the Minnesota spectra in mid-CBL, with a single peak at $z_{i}$ scale followed immediately by a true inertial subrange, tells us that all turbulence kinetic energy introduced into this flow is at the scale of the main convective circulations with none at larger wavenumbers. Our interpretation is that buoyant production of kinetic energy in the CBL, given by half the surface buoyancy flux, $g H_{0} / 2 T$, is overwhelmingly associated with the large organized thermals and subsidence regions of the main convective circulations. Smaller parcels of warmer air, which certainly exist, must be swept along within the main convective circulation and so not free to rise independently.

\subsubsection{Energy scaling}

This gives a new perspective on velocity scaling. The value of $z_{i} \epsilon_{o}$ is essentially the dissipation rate of turbulent kinetic energy integrated across the CBL, so the velocity scale $\left(z_{i} \epsilon_{o}\right)^{1 / 3}$ reflects the turbulence energy budget of the whole CBL. The surface buoyancy flux is not the only source term in this budget: turbulence energy is produced in the shear layer at the top of the CBL; it is lost as work done against drag at the ground; it is lost as work done against buoyancy during entrainment through the capping inversion; and turbulence kinetic energy can also be transferred internally from the mean flow to the fluctuations when wind blows over sharp topography (Smeets et al., 1998; Cava et al., 2005). All these processes contribute to the net production of turbulence energy in the CBL, and so contribute to the dissipation of that energy, which must balance the net production over time. The turbulence energy budget also has a rate of storage term, so there is usually a significant phase lag between buoyant production and dissipation. The dissipation rate depends on the scale and energy density of the turbulence in the CBL, both of which lag the buoyant production. This lag explains why the results from Minnesota were not correctly scaled by $w_{*}^{2}$ alone, even though buoyant production was the dominant production term in the overall energy budget, with $\psi$ values ranging about the value 0.5 expected when dissipation equals buoyant production. This is why the Minnesota procedure of matching inertial subranges is the correct procedure, even while the description of this scale as an adjusted Deardorff scale is misleading.

In more complex situations $\epsilon_{o}$ is even less directly related to the instantaneous rate of buoyant production. For example, Schneider and Lilley (1999) describe a CBL where a downwards flux of kinetic energy, presumably introduced by entrainment into that CBL, made a substantial contribution to its kinetic energy budget. Their "total energy production scaling velocity" included this and worked much better than the Deardorff scale in the lower two-thirds of their CBLs. In such cases $\left(z_{i} \epsilon_{o}\right)^{1 / 3}$ should still serve as the convective velocity scale, while the Deardorff scale will give erratic results.

At Minnesota it was found that $u$ and $v$ spectra observed nearer the ground, close above the surface friction layer, scaled in the same way as those observed in the middle of the CBL. That is to say, there was no evidence of any contribution of kinetic energy to the turbulence energy budget at local scales. In particular, it seems that buoyancy forces do not produce kinetic energy at local scales. This assessment parallels a similar conclusion reached by McNaughton (2006) for the unstable surface friction layer. Thus there is a consistent case that local buoyancy forces are opposed by local pressure reaction forces, which pressures refer the energy upscale, eventually to the scale of the main circulations in the CBL, and that this energy, net of the various other sources and sinks in the CBL, provides the power for the turbulent 
motions in the whole CBL. Since buoyancy makes no local contribution to the local kinetic energy balance at finer scales, the layer where $|L|<z \ll z_{i}$ is not a local free convection layer but is that part of the CBL flow that lies above the surface friction layer but near enough to the ground that significant eddies of the outer inertial subrange are strongly affected by blocking at the ground. Hunt and Morrison (2000) call this the "shear surface layer" in their model but, as we have seen, shear is not a significant factor in determining the form of turbulent spectra here, and their linearized analysis produces results that are incompatible with the Minnesota spectra.

\subsection{Scaling the $w$ spectra}

The impinging-eddies model for the lower part of the outer layer of CBLs also provides a velocity scale for $w$ spectra. Outer eddies impinge onto the ground, as we have argued, and are blocked by it. At levels where $z \ll z_{i}$ blocking forces the motion within eddies with lenghts comparable to $z_{i}$ to be almost horizontal. Such eddies contribute very little to the $w$ spectrum and so $z_{i}$ is not a relevant scaling length for the main part of this spectrum. When $z \ll z_{i}$ the wavenumbers of the eddies contributing most to the vertical velocity spectrum fall within the inertial subrange of the outer spectrum. This subrange has no intrinsic length scale, so we must look for one elsewhere. We expect the eddies that contribute most effectively to the $w$ spectrum to have heights roughly twice the observation height, so $z$ should scale the peak wavenumber. Eddies from the outer inertial subrange have energies that scale as $\left(\epsilon_{o} / \kappa\right)^{2 / 3}$, so eddies of height $\sim$ have energies proportional to $\left(z \epsilon_{o}\right)^{2 / 3}$, which provides the energy scale we seek for $w$ spectra near the ground. The corresponding velocity scale is $\left(z \epsilon_{o}\right)^{1 / 3}$, not the "local free convection" velocity scale $u_{f}$. Notice that these are not just dimensional results: Kolmogorov's spectral law for the outer inertial subrange gives both the $\epsilon_{o}$ parameter and the $2 / 3$ power for this energy scale. Dimensional consistency is just a necessary adjunct.

This new interpretation of the Minnesota results implies that the transition from the surface friction layer up into the outer layer is not a transition from a layer where buoyancy has no local effect to one where local buoyancy effects dominate, but a transition between two layers each with local dynamics that are dictated by flow in the whole CBL.The local buoyancy forces must power the outer convection, but they have their effect at the aggregated scale of the main circulations. The individual plumes do not rise independently but are constrained by pressure forces to be consistent with the turbulence energy cycle in the whole CBL.

\section{Experimental}

Turbulence measurements were made over an extensive playa surface at the SLTEST site in Western Utah from 23 May to 3 June 2005. Results reported here are from an array of eighteen North-facing CSAT3 sonic anemometers (Campbell Scientific Inc.). Nine of these were mounted on a tower at $1.42,2.14,3.00,4.26,6.14,8.71,12.5,17.9$, and $25.7 \mathrm{~m}$ above ground. The other nine were mounted on tripods in a cross-wind line, set variously at $3.00 \mathrm{~m}$ height with $10-\mathrm{m}$ spacing, $2.14 \mathrm{~m}$ height with $10-\mathrm{m}$ spacing or $2.14 \mathrm{~m}$ height with $3-\mathrm{m}$ spacing. This horizontal array was installed principally to serve another experiment but we use this data, averaged across the six Western-most anemometers, to provide the site heat flux, $H_{0}=\overline{w^{\prime} T^{\prime}}$, and averaged spectra to crosscheck the tower spectra at one level.

Precipitation had been higher than usual in the seasons preceding the experiment, resulting in a high water table, a very smooth surface and an energy balance that favored a basin circulation bringing winds from the North, in which direction the surface was uniform for about $100 \mathrm{~km}$. The site is described by Klewicki et al. (1995). Figure 1 shows the site and the array of sonic anemometers.

All the CSAT3 anemometers had been factory-calibrated in still air in the year preceding the experiment, most in the month preceding the experiment. Data were collected at $20 \mathrm{~Hz}$. Sampling was synchronized across the entire array using three CR5000 data loggers (Campbell Scientific) connected in a master-slave configuration. Thus the wider experimental design allowed for analysis of turbulent structures passing through the entire array.

A crane was used to access the tower and mount the CSAT3 anemometers. This allowed us to level them to within $\pm 0.5^{\circ}$.

The playa was very wet at the time the tower was erected, so trucks could not drive out onto its soft surface. As a result the tower was erected close to the raised berm where our trailers were parked (Fig. 1). The berm surface was $0.8 \mathrm{~m}$ above the playa surface, and our tower was erected 6 meters from its North-West corner. This caused some flow distortion at the tower. Sonic anemometers in the horizontal array at $10 \mathrm{~m}$ or more West of the tower gave very uniform results, but comparisons between these and instruments on the tower at the same level showed that mean wind speed at $3 \mathrm{~m}$ on the tower was reduced about $6 \%$ for Northerly winds. Comparisons of spectra show much smaller effects, being insignificant in the results reported here.

\section{Data selection and processing}

We selected 69 runs of half an hour duration, each from a highly-convective period when winds were from the North and the modulus of the Obukhov length, $|L|$, was less than $2 \mathrm{~m}$. This means that the depth of the surface friction layer, 


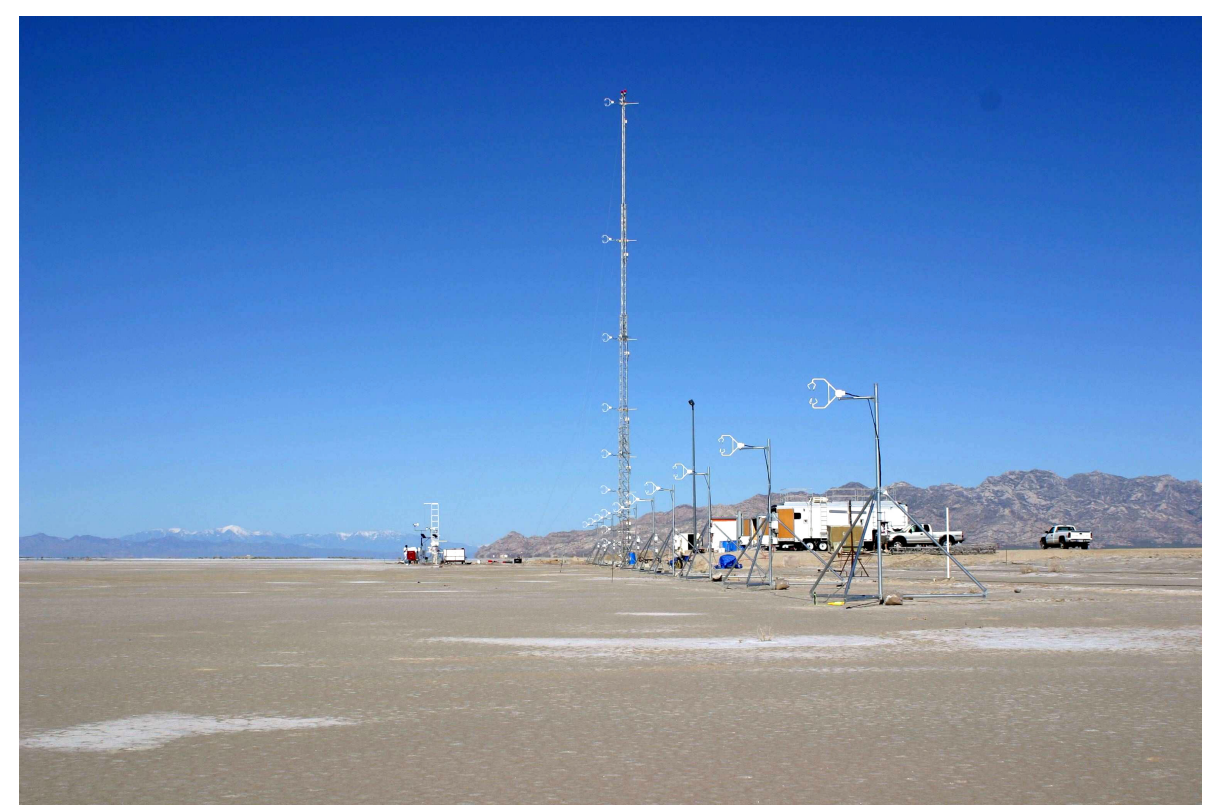

Fig. 1. Looking East at the array of CSAT3 sonic anemometers at the SLTEST site in May, 2005. The instruments were placed forward of a raised causeway (where the vehicle on the right is parked) and berm (where the trailers are parked), giving about $100 \mathrm{~km}$ of unobstructed fetch for winds from Northerly directions. Beyond the sonic array, on the playa, is a 5-m tower and two instrument trolleys. These were used in other experiments.

$z_{s}$, was always less than about 4 meters, assuming $z_{s} \sim 2|L|$ (McNaughton, 2004b). For these runs, at least the top six sonic anemometers were above the surface friction layer. All anemometers lay well within the bottom tenth of the boundary layer, assuming $z_{i}>500 \mathrm{~m}$.

Because of the extreme flatness and uniformity of the site we expected mean winds to blow horizontally. We rotated our coordinate system horizontally to set the $x$ direction into the mean wind for each run. Other rotations were unnecessary since the instruments were accurately leveled. Even so, the calculated wind elevation angles for half-hour periods were significantly different to zero: winds along the instrument axis had apparent elevations of $1.5^{\circ}$, and elevations varied systematically with wind direction. The pattern was similar for all 18 instruments, regardless of location. We interpret these non-zero elevation angles as instrumental artifacts. The results and interpretations presented below are robust with respect to this uncertainty. We calculated temperature spectra using the sonic virtual temperature (based on air density) from the CSAT3 anemometers.

We used Taylor's frozen-turbulence hypothesis to convert from frequency to wavenumbers in all our results, using $\kappa=2 \pi f / U_{25.7}$ where $f$ is frequency and $U_{25.7}$ is the mean wind speed at our top level at $25.7 \mathrm{~m}$. Taylor's frozenturbulence assumption is problematic because it cannot properly accommodate coherent eddies in sheared flows. We assumed that the turbulence moves as a single frozen block, and used wind speed at our highest level, $U_{25.7}$, as our best estimate of the speed of that block. This correctly registers the main peaks in the $u$ and $v$ spectra observed at the various heights. Even so, we expect very small eddies to travel with the local wind at each height, so we should use $\kappa=2 \pi f / U_{z}$ to calculate wavenumbers where $\kappa z>1$. For the runs selected here the average ratio $U_{25.7} / U_{1.42}$ was only 1.25 , so distortions to our results can be neglected to within that tolerance.

For each run we calculated the scaling parameter $\epsilon_{o}$ from the $u$ spectra. Fig. 2 shows that the averaged $u$ spectrum at $25.7 \mathrm{~m}$ follows the $-2 / 3$ reference line for $\kappa$ in the range $0.3 z<\kappa<0.2 f_{o}$, where $f_{o}$ is the Nyquist frequency (Kaimal et al., 1968), and the averaged $u$ spectrum at $1.42 \mathrm{~m}$ follows it in the range $20 / z_{i}<\kappa<400 / z$. We therefore fitted $-2 / 3$ reference lines to log-log plots for these parts of the top and bottom $u$ spectra. Values of $\epsilon_{o}$ were then obtained from Kolmogorov's law, which can be written as

$\kappa^{5 / 3} S_{u}(\kappa)=\alpha_{1} \epsilon_{o}^{2 / 3}$

We assumed the Kolmogorov constant, $\alpha_{1}$, to be 0.50 , as recommended by Sreenivasan (1995). The final values of $\epsilon_{o}$ so obtained are more strongly influenced by the upper $u$ spectrum than the lower one because the upper spectrum has more spectral data points in its chosen range. To a first approximation, the calculated values of $\epsilon_{o}$ are those for the true inertial subrange of the upper spectrum. The inversion height for each run was estimated using the Minnesota relationship, $z_{i}=\lambda_{m} / 1.5$ where $\lambda_{m}$ is the wavelength of the maximum of the pre-multiplied $u$ spectrum at $25.7 \mathrm{~m}$. 


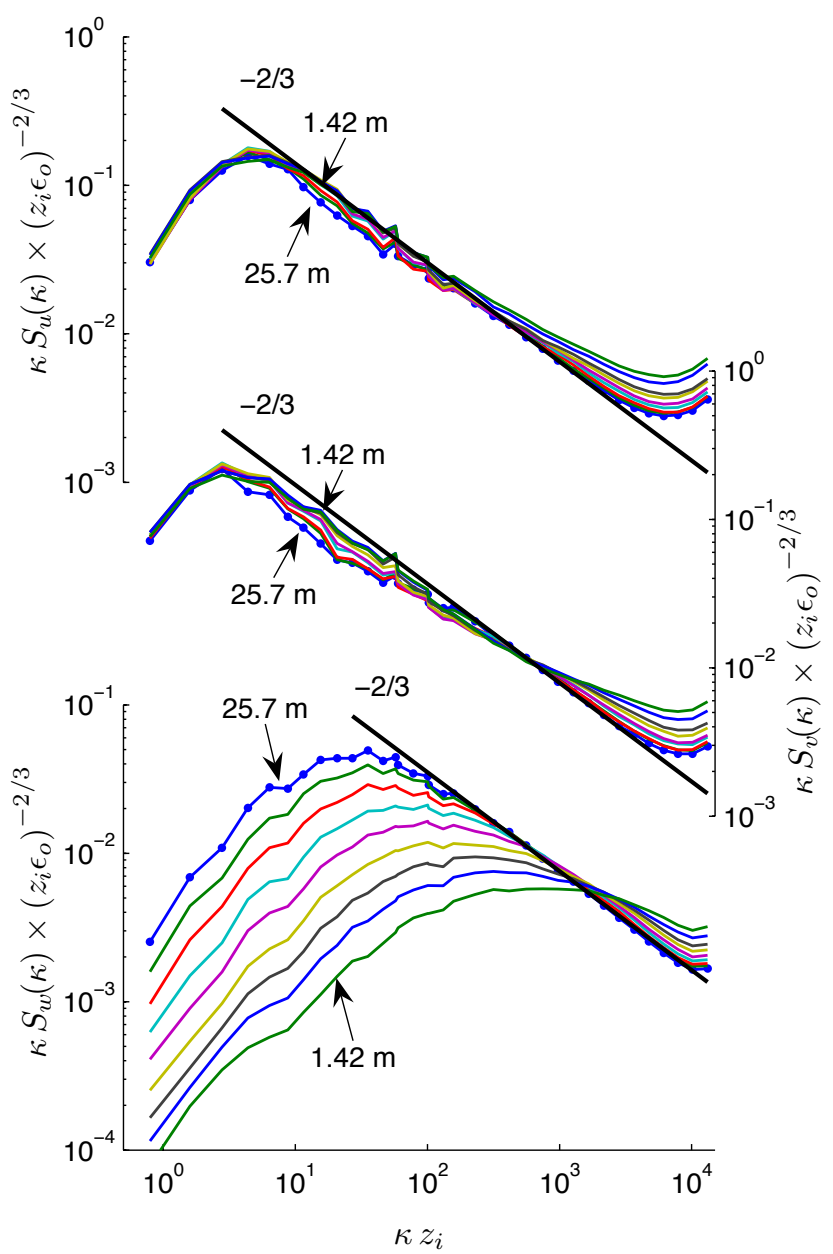

Fig. 2. Pre-multiplied power spectra for $u$ (top), $v$ (middle) and $w$ (bottom) components of the wind averaged for each of the 9 levels on the tower at the SLTEST site. All data are from unstable conditions with $|L|<2 \mathrm{~m}$, where $L$ is the Obukhov length. Results are plotted on outer-scaled axes (see text) so the $u$ and $v$ spectral peaks for each level are in the same position. The line marked $-2 / 3$ indicates the Kolmogorov law for inertial subranges. Color coding is indicated on the bottom plot, with the anemometer levels in sequence from highest at $25.7 \mathrm{~m}$ (blue with dots) down to lowest at $1.42 \mathrm{~m}$ (green). The top level is marked by dots.

Thus we used single values of $\epsilon_{o}$ and $z_{i}$ for scaling all spectra from each run. Scaled spectra were then averaged across all runs. This gave 9 sets of spectra: one set from each level. Since spectra from all levels were scaled using the same run parameters, any differences found in spectra from different levels are direct expressions of the height variations displayed in the raw data, with no artifacts introduced by different scaling at different levels.

\section{Results and discussion}

We deal with the velocity spectra first, then go on to the temperature spectra. Cospectra and other results not directly concerned with the scaling properties of spectra are held over until a later paper.

\subsection{Velocity spectra}

Averaged velocity spectra from the SLTEST 2005 experiment are shown in Fig. 2. Also shown are the $-2 / 3$ reference lines, representing Kolmogorov's law for inertial subranges. In general terms our results are similar to those reported from the Minnesota experiment (Kaimal et al., 1976). However, our spectra also display systematic deviations from the Minnesota "reference spectra". We will focus on these differences as a test of our interpretation of the Minnesota results.

\subsubsection{The $u$ and $v$ spectra}

Of the spectra in Fig. 2, the $u$ spectra are the easiest to analyse because they are free of the aliasing effects suffered by cross-wind components. A $-2 / 3$ line representing the outer inertial subrange can be easily and accurately fitted by eye to these $u$ spectra, as shown, but spectra from the various levels show systematic deviations about this line. At smaller wavenumbers, around $\kappa z_{i}=20$, the lowest $u$ spectrum follows the reference line quite well while spectral energies at other levels reduce progressively as heights increase: by $25.7 \mathrm{~m}$ the energy here is about $1 / 4$ less than the reference line. At larger wavenumbers, around $\kappa z_{i}=10^{3}$, the situation is reversed: the top spectrum follows the reference line quite well while the lower spectra become progressively more energetic as height decreases. That is, there is "extra" energy in each spectrum at larger wavenumbers and "missing" energy at smaller wavenumbers. Notice that these are relative to the reference spectrum: the total amount of spectral energy decreases systematically with height in both wavenumber ranges.

The $v$ spectra behave in a similar way, though upper and lower segments here do not line up so well along the $-2 / 3$ line. We attribute this to different amounts of aliasing in the two spectral ranges. Aliasing occurs because cross-wind spectra along a streamwise transect through three-dimensional velocity field contain, at each wavenumber, contributions from smaller wavenumbers of the threedimensional field (Pope, 2000). Aliasing shifts the spectra of lateral ( $v$ and $w$ ) components in isotropic turbulence upwards in wavenumber in the ratio $4 / 3$, but has no effect on stream-wise spectra (Monin and Yaglom, 1971). Wavenumber shifts for non-isotropic turbulence are unknown but the evidence of our $v$ spectra is that aliasing is less important at smaller wavenumbers where the motions are almost horizontal. The energy in the $u$ and $v$ spectra near peak wavenumbers does not change with height, as expected from the Minnesota 
results, giving confidence that the differences found at larger wavenumbers are real.

Overall, the $u$ and $v$ spectra in Figs 2 are broadly consistent with the reference spectra based on results from Minnesota, but they differ in detail. We now consider those differences.

\subsection{2 "Extra" energy at larger wavenumbers}

Compared to the spectra at the top of the mast, the scaled $u, v$ and $w$ spectra at lower heights all display "extra" energy at larger wavenumbers, with the amount of extra energy increasing towards the ground (Figs. 2 and 3). This progression remains when we ignore the upward hooks at the largest wavenumbers, which are caused by the aliasing associated with the sampling rate. These parts of the spectra cannot be collapsed by rescaling since the curves are not parallel. We expect to see extra energy at the lowest three anemometer since the surface friction layer would sometimes have grown up to engulf them. They would then have directly sampled the extra turbulence kinetic energy to be found there, and this would be reflected in the averages. However, we found extra energy all the way up to $25.7 \mathrm{~m}$ - up to more than 6 times the estimated height of the surface layer - and this extra energy reduced systematically over the whole range, not showing any sign of a definite cut-off level. We conclude that the excess energy must have been lifted from the surface layer where it was produced and transported up to various higher levels by an organized turbulence process.

This explanation is compatible with what is known of the kinetic energy budget of the surface friction layer. McNaughton (2006) modeled the kinetic energy budget of that layer by noting that it is driven from above by both the mean wind and the large eddies acting horizontally at the top of the friction layer. A problem with this model was that it could not explain why the dissipation rate (calculated from the kinetic energy at fine scales) in the lower part of the surface friction layer $(0<-z / L<0.5)$ is typically smaller than the neutral value, $u_{\epsilon}^{3} / \mathrm{kz}$. It now appears that outer eddies extend down to the ground and lift fine-scale eddies from the surface friction layer up to higher levels. McNaughton was therefore wrong to neglect the cross-scale interaction term $\overline{\tilde{w} e^{\prime \prime}}$ in his kinetic energy budget for the surface layer, were $\tilde{w}$ is the fluctuating vertical velocity associated with the outer eddy motion and $e^{\prime \prime}$ is the fluctuating kinetic energy associated with the shear-created inner eddies. This interpretation is supported by the work of Khalsa (1980) who found that periods of more than a few seconds with enhanced kinetic energy at fine scales can be used to identify "active plumes" in the surface friction layer.

The energy that McNaughton found to be "missing" from the surface friction layer is thus found higher up in the profile, transported there as the energy of the fine-scale eddies embedded in the larger-scale outer structures. The surface shear processes thus act within the lowest parts of the outer eddies, not beneath them as proposed by McNaughton (2006). The surface friction layer is then not a distinct layer but an average over local events occurring in the bottoms of many large eddies. This revised model opens a way of explaining why scalars statistics and velocity statistics scale differently through most of the surface friction layer. This is possible if, through most of the surface friction layer, the main motion of the outer large eddies transports scalars more efficiently than the smaller-scale but more energetic motions of the momentum-transporting structures (TEAL structures in the model of McNaughton (2004b)) within them. Such an explanation would solve the problem of different scalings for velocity and scalar statistics, as discussed by Kader and Yaglom (1990) and mentioned in our introduction, above.

The velocity spectra presented here give no direct information on the form of these outer eddies, apart from the 4.5:1 length-to-height ratio deduced from the velocity spectra, or how they might transport finer-scale eddies up from the surface layer. Experimental evidence from SLTEST 2005, to be presented in a later paper, makes it likely that they are pairs of counter-rotating stream-wise vortices, or perhaps alternating arrays of such vortices. Paired streamwise roll vortices have been deduced from field data using empirical orthogonal functions (Zhuang, 1995). Rotating eddies like this, with heights at least an order of magnitude greater than $z_{s}$, could sweep up the surface layer and carry it aloft in the form of sheet plumes, carrying with them heat, other scalars produced at the ground and embedded fine-scale eddies. The mechanism has been described by Mehta and Bradshaw (1988). We will discuss sheet plumes further, after we have introduced our temperature spectra in Sect. 5.3.

\subsection{3 "Missing" energy at smaller wavenumbers in $u$ and $v$ spectra}

The $u$ spectra at the lowest levels follow the $-2 / 3$ reference line very well, but in the range $8<\kappa z_{i}<300$ they fall progressively below that line as height increases (Fig. 2). The amount of energy "missing" increases with height, being about one quarter of the reference energy at $25.7 \mathrm{~m}$ and $\kappa z_{i} \sim 30$. The near-surface turbulence at these wavenumbers follows the reference spectrum even while the velocity spectra at greater heights does not. That is to say, information on the ideal form of this spectrum is somehow transmitted down from above by turbulence whose own velocity spectra do not observe the ideal form. The probable agent is pressure fluctuations, which were not measured. The $v$ spectra show similar behavior, though the upper spectra are off-set from the $-2 / 3$ line by different aliasing of the signals at small and large wavenumbers.

We argued above that the velocity scale should reflect the turbulence energy density of the flow, and so be based on $\epsilon_{o}$. If kinetic was the only kind of energy then we would expect velocity power spectra to collapse onto universal forms when scaled his way, but Figs. 2 and 3 do not show this. 


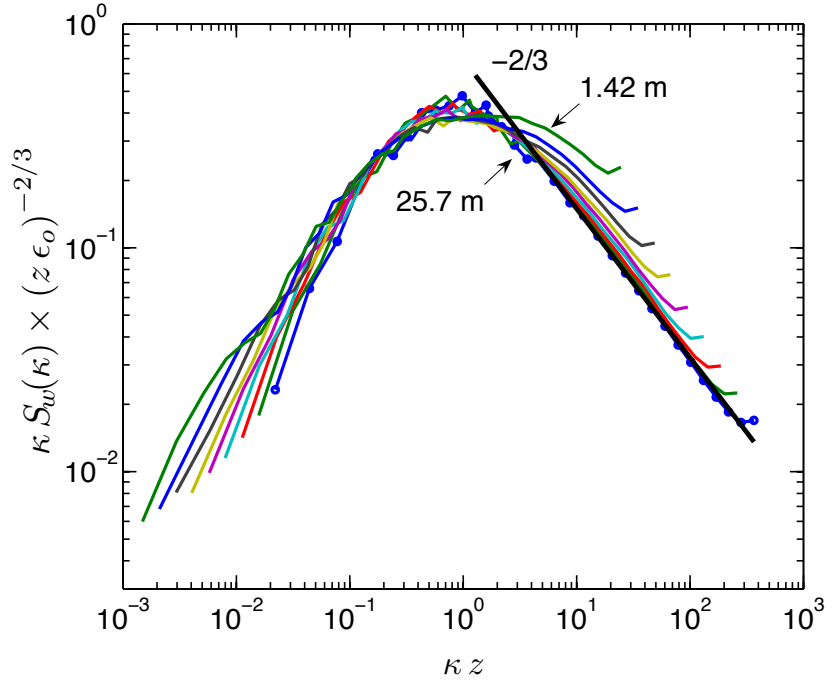

Fig. 3. Pre-multiplied spectra of vertical velocity fluctuations normalized using a velocity scale constructed from mixed-layer variables, $\left(z \epsilon_{o}\right)^{2 / 3}$, and plotted against $\kappa z$. Also shown is the $-2 / 3$ line showing the slope of predicted inertial subrange of the reference spectrum. Coding of levels is the same as in Fig. 2.

Our explanation is that kinetic energy is not the only kind of turbulence energy involved, and full collapse would be expected only if the spectra represent total turbulence energy, not just the kinetic component. McNaughton (2006) has argued that it is the sum of the fluctuating kinetic and pressure potential energies, $\left(e^{\prime}+p^{\prime}\right)$ that are locally conserved in equilibrium flows. Here $e^{\prime}$ is the deviation from the mean kinetic energy and $p^{\prime}$ is the fluctuation in pressure potential energy. This is Bernoulli's theorem applied to the fluctuations in equilibrium flows where total turbulence energy is uniformly distributed. Gravitational potential energy fluctuations associated with buoyancy need not be included in the calculations because in this highly-organized flow the effects of buoyancy are fully expressed through the pressure fluctuations. Pressure in this context is the organizing principle which opposes the independent rise of plumes and transfers their buoyant energy upscale, eventually to power the large convective structures that span the whole flow. Our suggestion is that the "missing" kinetic energy in Figs. 2 and 3 is not missing at all, but takes the form of pressure potential energy. This is not detected by sonic anemometers and so is not represented in these spectra.

We know of no previous work on spectra of static pressure at small wavenumbers in convective conditions, but the results of McNaughton (2006) can give some guidance. In the surface friction layer the turbulence energy budget shows that the tendency of buoyant air parcels to rise is opposed by pressure reaction forces, so buoyancy makes no contribution to the kinetic energy budget at local scale. In the present context, we may understand this as the main circulation opposing the rise of plumes in the subsidence regions while the horizontal flow near the ground sweeps them in towards the roots of the main thermal up-drafts. This transfers the gravitational potential energy of the plumes (buoyant energy) up to the scale of the main convective circulations. The pressures necessary to oppose plume rise increase with height according to the relationship

$p^{\prime}=\frac{g}{T} \int_{0}^{z} \theta^{\prime}(z) \mathrm{d} z$

Within thermal structures that are large compared to observation height the dependence of $\theta^{\prime}$ on height can be ignored and this becomes $p^{\prime}=g \theta^{\prime} z / T$, so the fraction of turbulence energy in the form of pressure potential energy increases in proportion to $z$ for $\kappa z \ll 1$. Since our instrument heights are in geometric progression, the pressure components of the large plumes should have parallel and equally spaced spectra when plotted on the logarithmic axis of Fig. 2. This is consistent with the observations, within experimental error.

\subsection{The $w$ spectra}

The $w$ spectra shown in Fig. 2 are also similar to those obtained by Kaimal et al. (1976) at Minnesota. However, and as expected from the discussion in Sect. 2.2, these spectra do not collapse when scaled using the outer length scale, $z_{i}$.

\subsection{1 w spectra at peak wavenumbers}

Good collapse of $w$ spectra is achieved when wavenumber is normalized using $z$, and spectral energy is normalized using $\left(z \epsilon_{o}\right)^{-2 / 3}$, as shown in Fig. 3. This is as predicted in Sect. 2.2 using the "impinging eddies" model. The spectral peaks are at $\kappa z \approx 0.7$, so the eddies contributing most to these spectra have wavelengths, $\lambda_{m}\left(=2 \pi / \kappa_{m}\right) \approx 9 z$. This compares with a $\lambda_{m} / z \approx 6$ from the Kansas and Minnesota experiments (Kaimal et al., 1972, 1976). These impinging eddies are therefore more elongated than the most energetic eddies of the frictional turbulence nearer the ground, for which $\lambda_{m} / z \approx 2$ (Kaimal et al., 1972).

Most of the energy in the $w$ spectrum is found near the peak, in which range the spectrum scales on $\left(z \epsilon_{o}\right)^{2 / 3}$, so the standard deviation of $w$ should scale quite well on $\left(z \epsilon_{o}\right)^{1 / 3}$. Figure 4 confirms this, but also shows the small increase at lower levels caused by the increase towards the ground of spectral energy at much larger and much smaller wavenumbers. We now look at these extremes.

\subsection{2 $w$ spectra at large wavenumbers}

At larger wavenumbers, beyond $\kappa z \sim 10$, the $w$ spectra show "extra" energy compared to the reference spectra, the amount decreasing with height. Similar behavior was observed in the $u$ and $v$ spectra, and we expect this "extra" energy also to represent the energy of the small eddies swept up from the surface layer. 

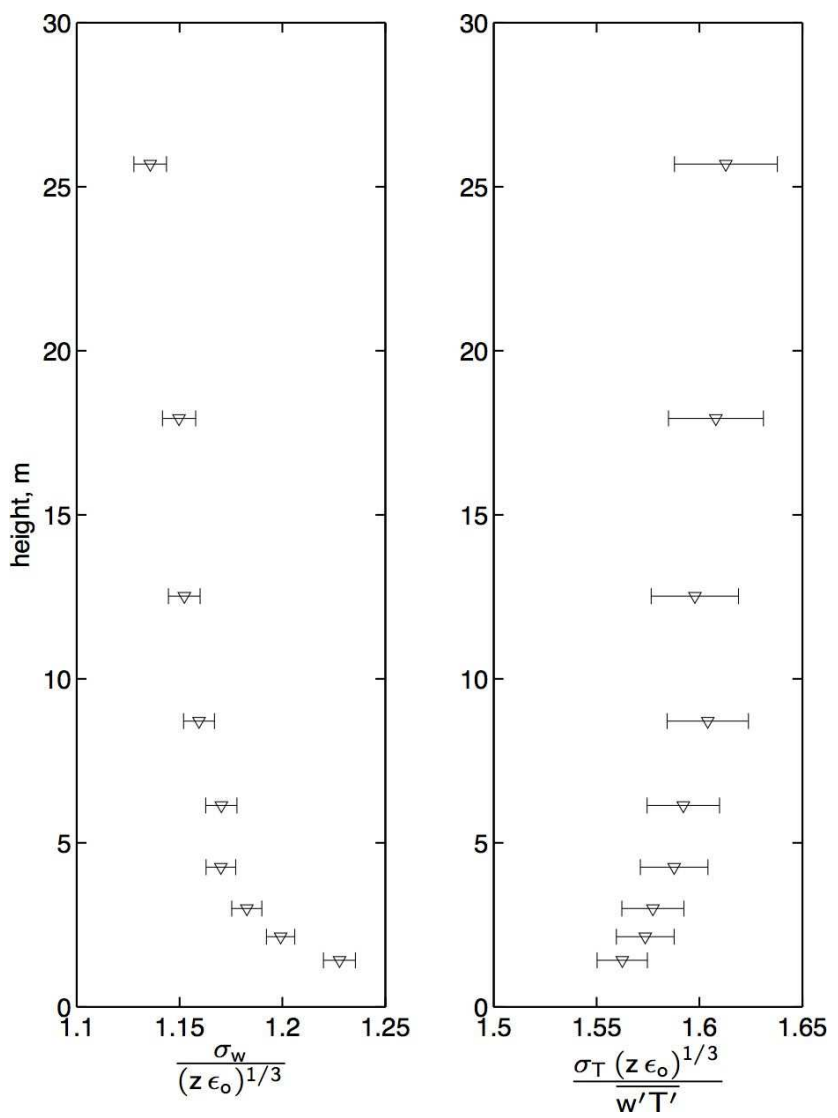

Fig. 4. Profiles of the standard deviations of vertical velocity, $\sigma_{w}$, in panel (a), and temperature, $\sigma_{T}$, in panel (b). Values are scaled as appropriate for eddies from the outer inertial subrange impinging onto the ground. Error bars indicate the standard errors of the estimates. Scaled $\sigma_{w}$ values decrease steadily with height as the extra contribution from the surface friction layer is left behind and turbulence energy is increasingly partitioned into the pressure fluctuations. Scaled $\sigma_{T}$ values increase slightly but progressively with height as a result of different scalings of the spectra for attached plumes, on the one hand, and detached temperature structures and fluctuations created by entrainment, on the other.

\subsection{3 $w$ spectra at small wavenumbers}

At smaller wavenumbers there is no reference spectrum, but spectral energy increases with height when scaled on $\left(z \epsilon_{o}\right)^{2 / 3}$, as shown in Fig. 3. Spectra there reflect the vertical velocities of the large, CBL-spaning eddies, with length scales comparable to $z_{i}$. These eddies have the energy scale $\left(z_{i} \epsilon_{o}\right)^{2 / 3}$, so we expect the kinetic energy in their vertical motions to reflect this scale, but reduced by the blocking effect of the ground. The $w$ spectra should therefore scale on $\left(z_{i} \epsilon_{o}\right)^{2 / 3}$ but also depend on $\left(z / z_{i}\right)$. There is no a priori reason why the $z / z_{i}$ dependence should be expressed as a power law, but empirical investigation shows that the pre-multiplied velocity spectra collapse well at small wavenumbers when normalized by $\left(z_{i} \epsilon_{o}\right)^{2 / 3} \times\left(z / z_{i}\right)^{4 / 3}$, as can be seen in Fig. 5 . Collapse is not so good for the lowest three levels, which sen-

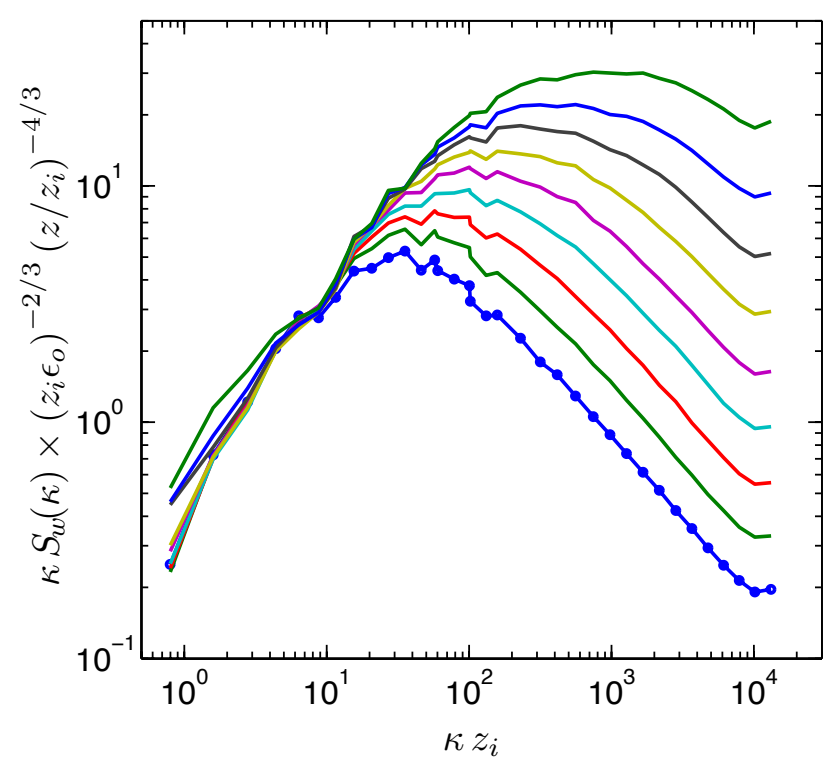

Fig. 5. Pre-multiplied $w$ spectra, normalized using $\left(z_{i} \epsilon_{O}\right)^{2 / 3} \times$ $\left(z / z_{i}\right)^{4 / 3}$ and plotted against $\kappa z_{i}$. The small-wavenumber parts of these spectra reflect the motions of large eddies which are blocked by the ground. These have velocity scale $\left(z_{i} \epsilon_{o}\right)^{1 / 3}$. Collapse is best achieved if the effect of blocking on vertical velocities is described by the empirical function $\left(z / z_{i}\right)^{2 / 3}$. The lowest three spectra are from levels near or sometimes within the surface friction layer and do not collapse so well onto the curve defined by the other levels. Coding of levels is the same as in Fig. 2.

sors may intermittently dip within the surface layer and so be directly affected by drag at the ground. We note that the vertical scale can also be written as $\left(z^{1 / 2} z_{i}^{1 / 2} \epsilon_{o}\right)^{2 / 3} \times\left(z / z_{i}\right)$. This form will be of interest when we consider temperature spectra at the same wavenumbers, below.

\subsection{The temperature spectra}

Pre-multipled temperature spectra for the same 69 runs are shown in Figs. 6 to 10. In Fig. 6 the temperature and wavenumber axes are scaled using as parameters $z_{i}, \epsilon_{o}$ and $H_{0}$. These spectra directly reflect the unscaled spectra, differing only by a constant multiplier on each axis. As at Minnesota, this scaling does not collapse the temperature spectra near the ground, but we note that it does register the positions of the subsidiary peaks near $\kappa z_{i} \approx 6$. Their positions are just where we found the peaks of the $u$-spectra in Fig. 2, so these minor peaks are associated with $z_{i}$-scale phenomena. The minor peaks also increase with height, so we identify them as a product of the entrainment of warmer air into the CBL from above. 


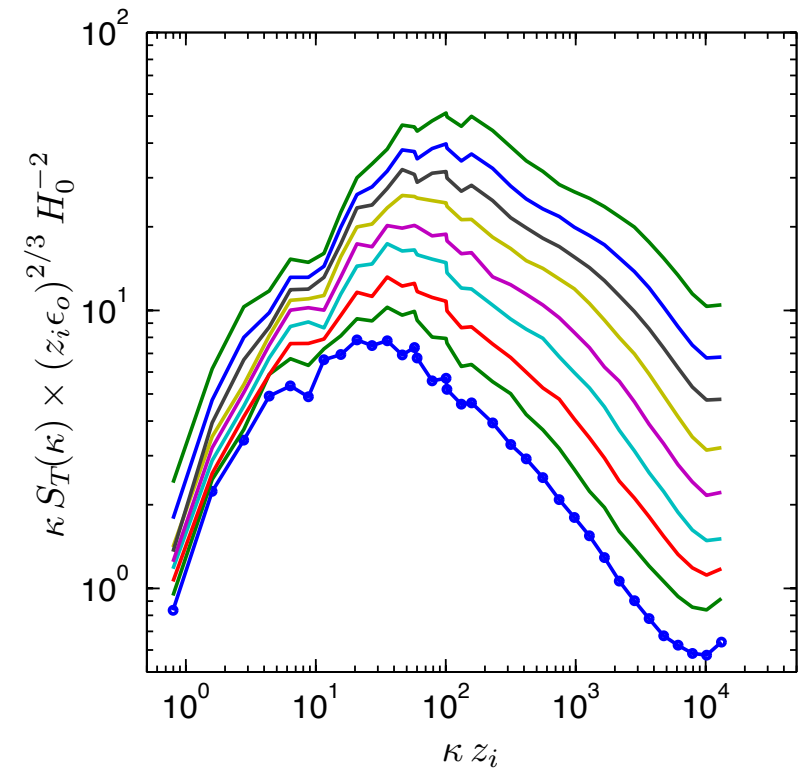

Fig. 6. Pre-multiplied spectra of temperature fluctuations. The spectra are scaled using $H_{0}^{2}\left(z_{i} \epsilon_{o}\right)^{-2 / 3}$ and plotted against $\kappa z_{i}$. This registers the position of the minor peak near $\kappa z_{i}=6$ but does not collapse the spectra.

\subsection{1 $T$ spectra at peak wavenumbers}

In Fig. 7 the spectra are scaled using the "impingement" scaling where $z$ replaces $z_{i}$ on both of the axis scales in Fig. 6 . This scaling is similar to "local free convection" scaling, but uses $\left(z \epsilon_{o}\right)^{2 / 3}$ in place of $u_{f}$. It is based on the same length and energy scales that were successful in collapsing the peaks of the $w$ spectra (Fig. 3), so we would expect this scaling to collapse temperature spectra if the streamwise lengths of the temperature plumes depend only on the energies and heights of the transporting eddies.

Figure 7 shows that only part of this expectation is fulfilled. Impingement scaling correctly normalizes the heights of the spectral peaks, so it does a good job of scaling the temperature variance (Fig. 4), but it does not register the positions of the spectral peaks. That is to say, vertical heat transport does depend on the energies of the transporting eddies, but something else helps determine the streamwise lengths of the plumes. Inspection of Figs. 6 and 7 suggests that an intermediate length scale $z^{\alpha} z_{i}^{1-\alpha}$ might be more successful. Empirical investigation finds best registration of the peaks when $\alpha=0.5$. This is shown in Fig. 8. This mixed length scale has not previously been reported.

The explanation for this mixed-length scaling seems to be that, though the eddies that carry the individual plumes have lengths proportional to $z$, the spectra reflect both these individual plumes and their coalescence into larger plumes in the flow. The coalescence must depend on $\left(z_{i} / z\right)$. Khanna and Brasseur (1998) describe the process thus: "Some small rather weak plumes near the ground are suppressed by

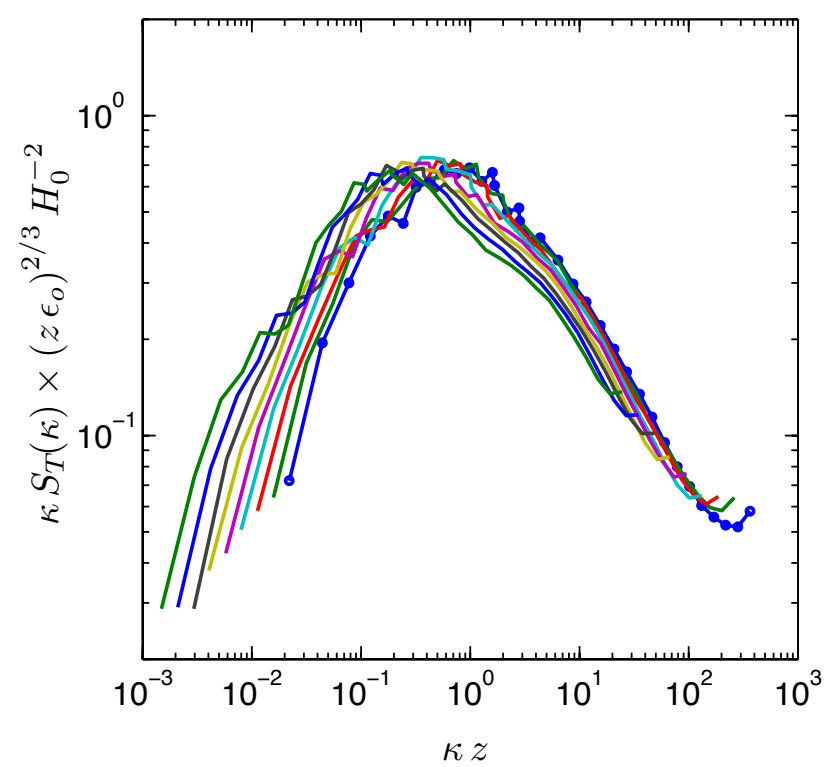

Fig. 7. Pre-multiplied temperature spectra, scaled using $H_{0}^{2}\left(z \epsilon_{o}\right)^{-2 / 3}$, and plotted against $\kappa z$. This scaling equalizes the heights of the main spectral peaks, but it does not put the peaks in the same position.

stronger downdrafts, while other plumes near the ground merge with their neighbors to form stronger updrafts". Thus the local plumes merge into larger-scale plumes, eventually to combine into the columnar thermals that reach up to the top of the CBL. For this aggregation to occur in a systematic way the positions of the $z$-scale eddies creating the plumes can not be random, but must be sensitive to the buoyancy of the plumes. That is, the convection in this layer is neither wholly free nor wholly driven from above, but rather that combination of the two where the local buoyant dynamics of the plumes find an accommodation with the energetics of the whole system. The mixed length scale of the temperature plumes thus reflects the local and large-scale controls acting together, as elements of a single self-organized system which transports heat efficiently upwards from the ground into the main part of the CBL.

The plumes that display this mixed scaling seem to have much in common with the plumes observed in laboratory studies and simulations of Rayleigh-Bénard convection (see, for example, the brief review and simulations by Schmidt and Schumann (1989)). The plumes take the form of elongated sheets of buoyant fluid rising from the surface and aligned with the local wind (Puthenveettil and Arakeri, 2005; Puthenveettil et al., 2005). "Wind" in the laboratory context is the component of the horizontal velocity near the wall that varies slowly on the time scale of the main circulations in the convection cells. In a convection experiment with an imposed cross flow, Theerthan and Arakeri (2000) found the sheet plumes to be aligned with the mean 


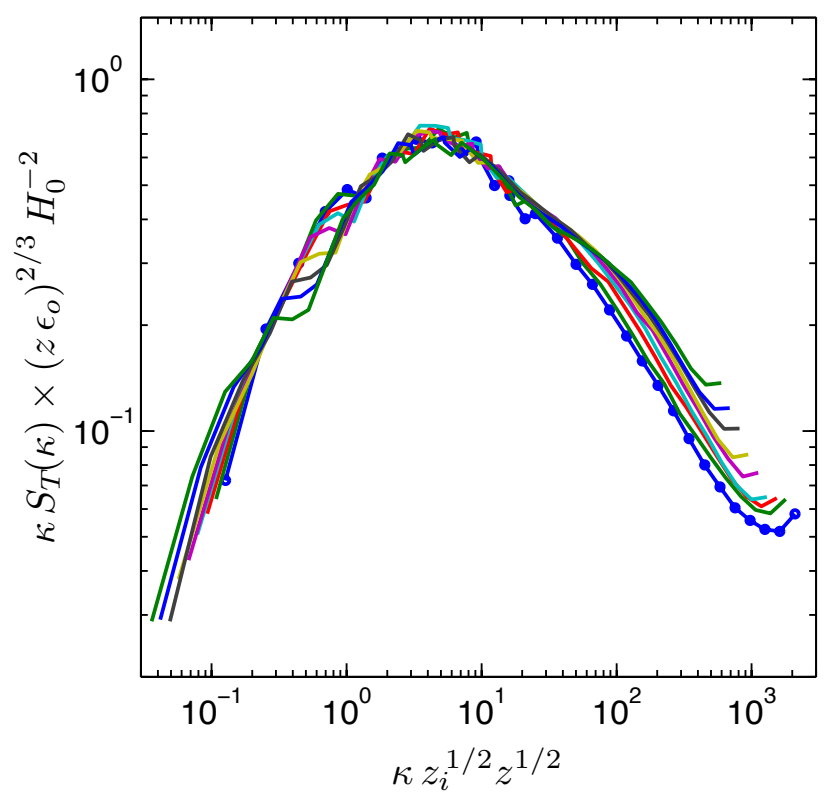

Fig. 8. Temperature spectra scaled as in Fig. 7, but with wavenumbers scaled using the mixed length scale $\left(z z_{i}\right)^{1 / 2}$. This scaling collapses these spectra in the region of the main peaks.

flow. These laboratory experiments were all done at Rayleigh numbers high enough to achieve "hard turbulence" but somewhat lower than those needed to make turbulent the friction layer against their heated lower plates. We propose that similar 'sheet plumes' form between pairs of counter-rotating roll vortices near the ground in CBLs. These are the same outer eddies that we appealed to above, when discussing $w$ spectra and the transport of fine-scale eddies up from the friction layer into the outer layer. Thus the roll vortices in contact with the ground sweep up air from near the ground, carrying aloft fine-scale eddies and scalars, such as temperature, embedded within it. Direct evidence that such structures occur outdoors comes from the thermal infra-red images of pasture land by Derksen (1974), which show linear temperature striations on the ground in windy, unstable conditions. Further evidence from the SLTEST site will be published separately.

Though this mixed-length scaling collapses positions of the spectral peaks rather well, collapse is poorer at smaller wavenumbers where $\kappa\left(z z_{i}\right)^{1 / 2}<5$ and at larger wavenumbers where $\kappa\left(z z_{i}\right)^{1 / 2}>50$.

\subsection{2 $T$ spectra at small wavenumbers}

At smaller wavenumbers the spectra separate into a set of subsidiary peaks with amplitudes that decrease with instrument height (Fig. 4). These eddies have characteristic height scale $z_{i}$, and so energy scale $\left(z_{i} \epsilon_{o}\right)^{2 / 3}$. We would therefore expect to find a spectrum that scales on $H_{0}^{2}\left(z_{i} \epsilon_{o}\right)^{-2 / 3}$ at mid levels in the CBL. Nearer the ground we would expect to see an effect of blocking by the ground, so the characteristic

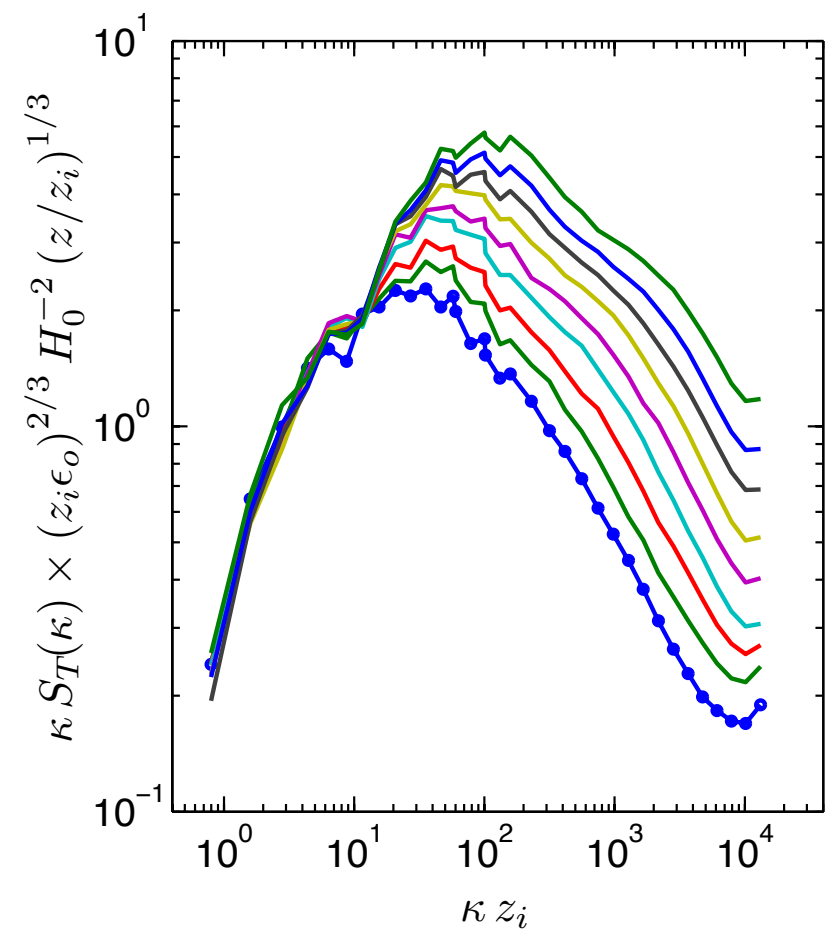

Fig. 9. Temperature spectra with wavenumbers scaled as in Fig. 6, but with the temperatures scaled on $H_{0}^{2}\left(z_{i} \epsilon_{o}\right)^{-2 / 3}\left(z / z_{i}\right)^{-1 / 3}$. This scaling collapses the spectra at very small wavenumbers, which reflects eddies whose sizes are limited by the height of the capping inversion at $z_{i}$.

vertical velocities should also to depend on $z / z_{i}$. Empirical investigation shows that scaling on $H_{0}^{2}\left(z_{i} \epsilon_{o}\right)^{-2 / 3}\left(z / z_{i}\right)^{-1 / 3}$ collapses the heights of these peaks quite well, as shown in Fig. 9.

The above argument is top-down, beginning with the energy of the large eddies. We can also take a bottom-up view in which the large eddies near the ground are formed by the coalescence of plumes into structures of scale $z_{i}$. The vertical velocities of the individual plumes would scale on $\left(z \epsilon_{o}\right)^{2 / 3}$ if they depended only on the vertical velocities of randomlychosen eddies, but as the plumes coalesce they increasingly become part of the large-scale structure of the CBL, and so are increasingly affected by the large-scale vertical motions in the CBL.

We note that the energy scale for the vertical velocity in Fig. 9, $\left(z_{i} \epsilon_{o}\right)^{2 / 3}\left(z / z_{i}\right)^{1 / 3}$, can also be written as $\left(z_{i}^{1 / 2} z^{1 / 2} \epsilon_{o}\right)^{2 / 3}$, revealing a mixed velocity scale like that which scaled the vertical velocity spectrum at small wavenumbers (Sect. 5.2.3) but lacking the factor $\left(z / z_{i}\right)$. The reason for this factor is obscure, but we note that temperature spectra reflect the distribution of the streamwise lengths of the plumes, so perhaps we should not expect this velocity scale to be the same as that which scales the energy of the vertical motions. 


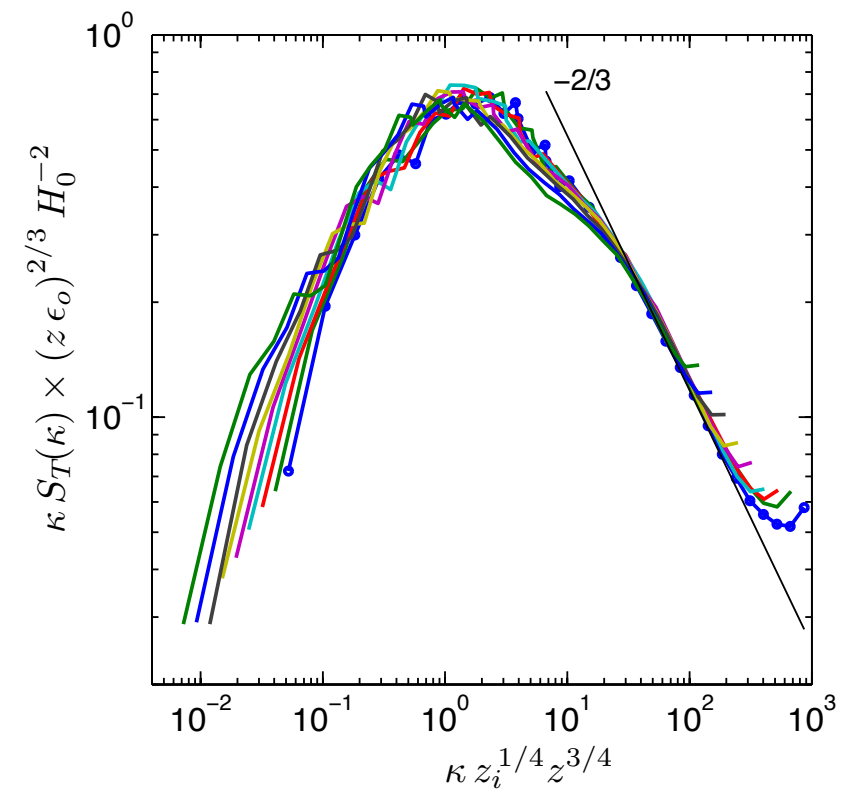

Fig. 10. Temperature spectra with wavenumbers scaled by the mixed length scale $\left(z_{i}^{1 / 4} z^{3 / 4}\right)$. This collapses the spectra at large wavenumbers, where the eddies are detached .

Mixed velocity scales are not common in fluid mechanics, but such a scale has been found to collapse near-wall peaks of $u$ variance near the tops of viscous sublayers over a flat plate in a wind tunnel (DeGraaff and Eaton, 2000) and outdoors (at the SLTEST site) in neutral conditions (Metzger, 2006). Analogy with the present case suggests that this may also reflect a mutual accommodation of the structural requirements of momentum transport by viscous and Reynolds stresses.

\subsection{3 $T$ spectra at large wavenumbers}

The scaling used in Fig. 8 does not collapse temperature spectra well at larger wavenumbers: when $\kappa\left(z z_{i}\right)^{1 / 2}>50$ or, alternatively, when $\kappa z>4 \pi$. Eddies in this size range are necessarily detached, so this part of the spectrum reflects the size distribution and arrangement of thermal filaments created by stretching and folding of the plumes as they break-up into smaller structures not directly rooted at the ground. Inspection of Figs. 7 and 8 suggests that still another length scale might collapse these spectra rather well - this one intermediate to the simple $z$ scale of Fig. 7 and the mixed $\left(z z_{i}\right)^{1 / 2}$ scale of Fig. 8. Figure 10 demonstrates the collapse for this spectral range obtained using $\left(z_{i}^{1 / 4} z^{3 / 4}\right)$ as the length scale. The universal curve follows a $-2 / 3$ power law, as predicted by the Obukhov-Corrsin (Corrsin, 1951) extension of Kolmogorov spectral model to scalars in an inertial subrange. Spectra from even the lowest levels collapse onto this same curve, even though the $w$ spectra in this range can not be collapsed simply by scaling. "Extra" temperature variance is not transported upwards from the friction layer, even though velocity variance of this scale is so transported. The lack of transported variance in the $T$ spectra is consistent with the observation that temperature spectra obey outer scaling down to $-z / L \approx 0.04$ (Kader and Yaglom, 1990), which is always less than $10 \mathrm{~cm}$ from the ground for our 69 runs. Thus almost no "extra" temperature variance is produced near the ground, so almost none is available to be swept up to higher levels by the larger eddies. This mixed length scale must have another explanation.

A plausible explanation is that the $\left(z_{i}^{1 / 4} z^{3 / 4}\right)$ length scale reflects the breaking up of plumes having length scale $\left(z_{i} z\right)^{1 / 2}$ by a mechanism that depends only on $z$. That is to say, the large-scale organization which affects the lengths of the intact plumes does not reach down to further constrain the sizes or arrangement of the fragments of plumes. If this argument were correct the kinetic energy of the dispersing eddies would have to scale simply on height, which is not true of the results shown in Fig. 2 at these wavenumbers. However, we have proposed that the "extra" velocity variance shown in Fig. 2 represents the energy of small eddies swept up within the interiors of temperature plumes, so while these interior eddies may cause fine-scale mixing within plumes, they do not contribute to the break-up of plumes. The eddies whose decay is likely to affect the plumes most strongly are the very eddies associated with the formation of the plumes themselves, and these attached roll eddies do scale simply on $z$, as their $w$ spectra in Fig. 3 indicate.

\section{Conclusions and general discussion}

This paper has approached velocity scaling in a new way. In the past, scaling parameters were selected by combining, in various proportions, empirical information and intuition based on contemporary understanding of the particular flow. This understanding was based on interpretations of the terms of Navier-Stokes equations in their differential form. Once introduced, the chosen scaling parameters then gained general acceptance through their ability to reduce further experimental results to universal values or relationships. The friction velocity, $u_{*}$, and the Deardorff convective scale, $w_{*}$, were derived and became accepted in just this way. By contrast, we have approached velocity scaling through the energy budget of the turbulence. In effect we have reversed the usual procedure of first choosing a velocity scale then using it to scale the terms of the turbulent energy budget to one where we first create an energy scale, based on the turbulence energy budget of the whole flow, then use this to scale the local turbulence kinetic energy component of that budget. An implicit feature of our approach is that scaling is based on integral properties of the flow rather than the differential (local) properties. In this we break with the tradition that began with Richardson, continued on through the work of Prandtl, Obukhov and Deardorff, amongst others, and remains current to the present day. 
Our approach to length scales is more traditional. We find these empirically, and interpret them in terms of the lengths of the eddies or temperature structures in the flow. Empiricism is appropriate here because we still have much to learn about the nature and sizes of the eddies and, particularly, the thermal structures, their aggregations and their interactions or accommodations. While the length scales for our velocity spectra are consistent with past work, we find length scales for the temperature structures that are both unprecedented in their general nature and highly informative when interpreting the nature of thermal convection in CBLs.

Our particular conclusions about the scaling properties of spectra at levels where $z_{s}<z \ll z_{i}$ are:

1. The energy scale for the principal, large-scale motions in CBLs is $\left(z_{i} \epsilon_{o}\right)^{2 / 3}$. Here the outer dissipation rate, $\epsilon_{o}$, represents (balances) the net turbulence energy production in the whole CBL. This functionally replaces the Deardorff convective velocity scale.

2. The energy scale for the peak region of vertical velocity spectra is $\left(z \epsilon_{o}\right)^{2 / 3}$. The $-2 / 3$ power reflects the energy distribution in the outer inertial subrange. This scale functionally replaces the "local free convection" velocity scale.

3. The peaks of temperature spectra collapse when wavenumbers are scaled using the mixed length $z^{1 / 2} z_{i}^{1 / 2}$. This scale reflects both the length scale of the transporting eddies, $z$, and the organization of the plumes into convective structures which depend, in topdown fashion, on $z_{i}$.

4. At still larger larger wavenumbers, temperature spectra collapse when wavenumbers are normalized by the mixed length $z^{3 / 4} z_{i}^{1 / 4}$. This shows that small fragments, at length scales characteristic of detached eddies, are less organized than whole plumes.

To explain our results we propose two new hypotheses, which ammend and extend the structural model we have been developing in recent papers. These are:

1. Fine-scale eddies near the ground are swept upwards by the outer eddies, and above the surface friction layer their energy appears as "extra" energy in velocity spectra at small wavenumbers. For this to happen the outer eddies must reach down to the ground, and the shearproduced eddies be created within their lower parts as they scrape along the ground.

2. The $-2 / 3$ law for inertial subranges of premultiplied spectra applies to total turbulence energy, not just the kinetic component. Some turbulence energy exists in the form of fluctuating pressure potential energy. Because this is not represented in velocity spectra, some energy appears to be "missing" at smaller wavenumbers and greater heights when compared with the $-2 / 3$ power law associated with the outer inertial subrange. Pressure potential energy is associated with the pressure reaction forces needed to prevent the autonomous - "free" - rise of plumes and so maintain the self-organized, systemwide patterns of convection in CBLs.

Overall, we conclude that turbulent motions in CBLs are highly structured, and that this has a profound effect on the way we should model heat transport. The thermal structure of the CBL is self-organizing and, near the ground, it involves an accommodation between the requirements of the turbulent energy cascade and the buoyant rise of heatcarrying plumes from the ground. The plumes rise at rates which reflects the kinetic energy balance of the whole flow, not the local buoyancy of the plumes, so this is not "local free convection". The transition from an unstable surface friction layer to a convective outer layer is not, as suggested in the introduction, a transition from a layer where buoyancy has no local effect to one where local buoyancy forces wholly drive the local turbulent motions, but a transition between two layers in neither of which has buoyancy any direct local effect on the velocity scale. We conclude that the Obukhov length, which depends on the local buoyancy flux, is not a relevant parameter for describing this transition.

The present work suggests an answer to the question of why scalar statistics obey outer scaling to much lower levels than do purely velocity statistics. We have found that the outer eddies reach right down to the ground and the local frictional eddies, created by the outer eddies dragging along the ground, act within their lower parts. Our suggestion is that the outer eddies are much more efficient at transporting scalars within the surface friction layer than are the inner eddies that transport momentum there. That is, we agree with Lumley and Panofsky (1964) and Tennekes (1970) who argued that momentum and scalars must be transported by different kinds of eddies. Further results from the SLTEST 2005 experiment give considerable support to this proposition. We hope to present these in a later paper.

Finally, we make a general comment. Past scaling schemes have been based on a local view of turbulence, being based, in part, on the Navier-Stokes and thermodynamic equations in their differential (local) form. A great deal of progress has been made, as demonstrated by the successful applications of mixed-layer, "local free convection" and Monin-Obukhov similarity schemes to many practical tasks, but real problems remained. There has been residual scatter in scaled experimental results, and this scatter has not gone away despite more and better experiments (so that many accept that such variability is an irreducible property of atmospheric CBLs). There are systematic differences between laboratory and field results (such as the value of $\sigma_{w} / u_{*}$ being $\approx 1.0$ in wind tunnels but $\sim 1.25$ in near-neutral conditions outdoors), and these have had no obvious cause. Our scaling results, by contrast, reflect natural integrations of the 
governing equations, and our scaling parameters reflect the integral properties of CBLs: their net turbulence energy balances and their coherent structures. In our view CBLs are self-organizing systems in which the energy and organization of the whole flow reaches down to affect even the smallest parts (eddies or plumes) of the system. This approach promises better models of CBLs.

\section{Addendum}

Present results and past work allows us to propose a new scale length to replace the Obukhov length. This is the scale height of the transition from inner to outer layers. McNaughton (2004b) gave this as

$z_{s}=\frac{u_{*}^{3}}{k \epsilon_{o}}$

where $k$ is the von Kàrmàn constant. The term $u_{*}^{3} / k$ is related to the production of kinetic energy in the surface friction layer. In more recent work, McNaughton (2006) found that overhead convection, by varying the stength and direction of winds near the ground, increases the production of kinetic energy in the friction layer above that accounted by $u_{*}$ alone. To account for this he defined a 'dissipation velocity'

$u_{\epsilon}=\left(u_{*}^{3}+\overline{v_{*}^{3}}\right)^{1 / 3}$

where $v_{*}$ is the fluctuating counterpart of $u_{*}$ measured at the ground itself. $u_{\epsilon}$ then replaces $u_{*}$ as the velocity scale for the surface friction layer, so (5) becomes

$z_{s}=\frac{u_{\epsilon}^{3}}{k \epsilon_{o}}$

Transitions from the inner to the outer turbulence regime are then described by functions of $z / z_{s}$, not on $-z / L$, as in Monin-Obukhov similarity theory.

Acknowledgements. We thank J. Klewicki (U. Utah) for inviting us to participate in the SLTEST 2005 experiment. We thank the whole SLTEST team for their many contributions to the overall experiment, particularly J. Wilson (U. Alberta) and N. Hutchins (U. Minnesota) who helped collect the data used here. We thank E. Swiatek and B. Tanner of Campbell Scientific Inc. for assistance that went far beyond the terms of the contract when setting up the experiment. This work was supported by the U.K. Natural Environment Research Council under grant NER/A/S/2002/00958.

Edited by: P.-L. Sulem

Reviewed by: O. Cote and another referee

\section{References}

Cava, D., Schipa, S. and Giostra, U.: Investigation of low-frequency perturbations induced by a steep obstacle, Bound.-Lay. Meteorol., 115, 27-45, 2005.

Corrsin, S.: On the spectrum of isotropic temperature fluctuations in an isotropic turbulence, J. Appl. Phys., 22, 469-473, 1951.

Deardorff, W. J.: Preliminary results from numerical integrations of the unstable boundary layer, J. Atmos. Sci., 27, 1209-1231, 1970.

DeGraaff, D. B. and Eaton, J. K.: Reynolds-number scaling of the flat-plate turbulent boundary layer, J. Fluid Mech. 422, 319-346, 2000.

Derksen, W. J.: Thermal infrared pictures and the mapping of microclimate, Neth. J. Agric. Sci., 22, 119-132, 1974.

Holtslag, A. A. M. and Nieuwstadt, F. T. M.: Scaling the atmospheric boundary layer, Bound.-Lay. Meteorol., 36, 201-209, 1986.

Hunt, J. C. R. and Morrison, J. F.: Eddy structure in turbulent boundary layers, Euro. J. Mech. B D Fluids, 19, 673-694, 2000

Kader, B. A. and Yaglom, A. M.: Mean fields and fluctuation moments in unstably stratified turbulent boundary layers, J. Fluid Mech., 212, 637-662, 1990.

Kaimal, J. C. and Businger, J. A.: Case studies of a convective plume and a dust devil, J. Appl. Meteorol. 9, 612-620, 1970.

Kaimal, J. C. and Finnigan, J. J.: Atmospheric boundary layer flows, Oxford University Press, New York, 1994.

Kaimal, J. C. and Wyngaard, J. C.: The Kansas and Minnesota experiments, Bound.-Lay. Meteorol. 50, 31-47, 1990.

Kaimal, J. C., Wyngaard, J. C., and Haugen, D. A.: Deriving power spectra from a three-component sonic anemometer, J. Appl. Meteorol., 7, 827-837,1968.

Kaimal, J. C., Wyngaard, J. C., Izumi, Y., and Coté O. R.: Spectral characteristics of surface-layer turbulence, Q. J. Roy. Meteor. Soc., 98, 563-589, 1972.

Kaimal, J. C., Wyngaard, J. C., Haugen, D. A., Coté, O. R., Izumi, Y., Caughy, S. J., and Readings, C. J.: Turbulence structure in the convective boundary layer, J. Atmos. Sci., 33, 2152-2169, 1976.

Khalsa, S. J. S.: Surface-layer intermittency investigated with conditional sampling, Bound.-Lay. Meteorol., 19, 135-153, 1980.

Khanna, S. and Brasseur, J. G.: Three-dimensional buoyancy- and shear-induced local structure of the atmospheric boundary layer, J. Atmos. Sci., 55, 710-743, 1998.

Klewicki, J. C., Metzger, M. M., Kelner, E., and Thurlow, E. M.: Viscous sublayer flows at $R_{\theta}=1500000$, Phys. Fluids, 7, 857863, 1995.

Lumley, J. L. and Panofsky, H. A.: The structure of atmospheric turbulence, Interscience, New York, 1964.

McNaughton, K. G.: Attached eddies and production spectra in the atmospheric logarithmic layer, Bound.-Lay. Meteorol., 111, 118, 2004a.

McNaughton, K. G.: Turbulence structure of the unstable atmospheric surface layer and transition to the outer layer, Bound.Lay. Meteorol., 112, 199-221, 2004b.

McNaughton, K. G.: On the kinetic energy budget of the unstable atmospheric surface layer, Bound.-Lay. Meteorol., 118, 83-107, 2006.

McNaughton, K. G. and Brunet, Y.: Townsend's hypothesis, coherent structures and Monin-Obukhov similarity, Bound.-Lay. Meteorol., 102, 161-175, 2002. 
McNaughton, K. G. and Laubach, J.: Power spectra and cospectra for wind and scalars in a disturbed surface layer at the base of an advective inversion, Bound.-Lay. Meteorol., 96, 143-185, 2000.

Mehta, R. D. and Bradshaw, P.: Longitudinal vortices embedded in turbulent boundary layers Part 2. Vortex pair with "common flow" upwards, J. Fluid Mech., 188, 529-546, 1988.

Metzger, M.: Length and time scales of the near-surface axial velocity in a high Reynolds number turbulent boundary layer, Int. J. Heat Fluid Flow, 27, 534-541, 2006.

Monin, A. S. and Yaglom, A. M.: Statistical Fluid Mechanics: Mechanics of Turbulence, Vol. 1, English translation, edited by: Lumley, J. L., MIT Press, Cambridge, MA, 769 pp., 1971.

Pope, S. B.: Turbulent Flows: Cambridge, Cambridge University Press, 2000.

Priestley, C. H. B.: Free and forced convection in the atmosphere near the ground, Q. J. Roy. Meteor. Soc., 81, 139-143, 1955.

Puthenveettil, B. A. and Arakeri, J. H.: Plume structure in highRayleigh number convection, J. Fluid Mech., 542, 217-249, 2005.

Puthenveettil, B. A., Anathakkrishna, G., and Arakeri, J. H.: The multifractal nature of plume structure in high-Rayleigh-number convection, J. Fluid Mech., 526, 245-256, 2005.

Schmidt, H. and Schumann, U.: Coherent structure of the convective boundary layer derived from large-eddy simulations, J. Fluid Mech., 200, 511-562, 1989.
Schneider, J. M. and Lilly, D.K.: An observational and numerical study of a sheared, convective boundary layer. Part I: Phoenix II observations, statistical description, and visualization, J. Atmos. Sci., 56, 3059-3078, 1999.

Smeets, C. J. P., Duynkerke, P. G., and Vugts, H. F.: Turbulence characteristics of the stable boundary layer over a mid-latitude glacier. Part I: a combination of katabatic and large-scale forcing, Bound.-Lay. Meteorol., 87, 117-145, 1998.

Sreenivasan, K. R.: On the universality of the Kolmogorov constant, Phys. Fluids, 11, 2778-2784, 1995.

Tennekes, H.: Free convection in the turbulent Ekman layer of the atmosphere, J. Atmos. Sci., 27, 1027-1034, 1970.

Tennekes, H.: A model for the dynamics of the inversion above a convective boundary layer, J. Atmos. Sci., 53, 149-160, 1973.

Theerthan, S. A. and Arakeri, J. H.: Planform structure and heat transfer in turbulent free convection over horizontal surfaces, Phys. Fluids, 12, 884-894, 2000.

Webb, E. K.: Convection mechanisms of atmospheric heat transfer from surface to global scales, in: Proc. Second Australasian Conference on Heat and Mass Transfer, edited by: Bilger, R. W., University of Sydney, pp. 523-539, 1977.

Wyngaard, J. C., Coté, O. M., and Izumi, Y.: Local free convection, similarity, and the budgets of shear stress and heat flux, J. Atmos. Sci., 28, 1171-1182, 1971.

Zhuang, Y.: Dynamics and energetics of convective plumes in the atmospheric surface layer, J. Atmos. Sci., 52, 1712-1722, 1995. 